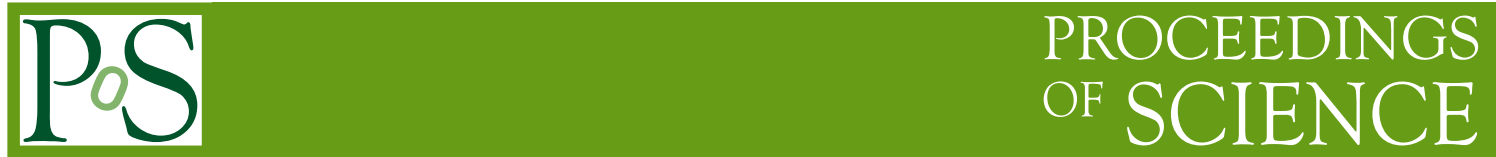

\title{
Probing dark matter with monojets in ATLAS at the LHC
}

\author{
Johanna Gramling* \\ University of Geneva \\ E-mail: jgramlin@cern.ch
}

A search for new phenomena in monojet final states with large missing transverse momentum was performed on $4.7 \mathrm{fb}^{-1}$ of data from proton-proton collisions at $\sqrt{s}=7 \mathrm{TeV}$, collected in 2011 with the ATLAS experiment at the Large Hadron Collider. Good agreement is observed between the number of events in data and the Standard Model prediction. The result is translated into limits on pair production of weakly interacting dark matter candidates.

Proceedings of the Corfu Summer Institute 2012 "School and Workshops on Elementary Particle Physics and Gravity"

September 8-27, 2012

Corfu, Greece

\footnotetext{
* Speaker.
} 


\section{Introduction}

Event topologies with a high-energetic jet and large missing transverse momentum present well-defined final-states, which are of interest for many physics models beyond the Standard Model (BSM) containing new stable, massive particles that escape the detector. A monojet signature can arise within the Standard Model (SM) from the production of a Z boson in association with a jet, where the $\mathrm{Z}$ boson decays into two neutrinos. Also the production of a $\mathrm{W}$ boson and a jet can be a background in case the charged lepton is not detected.

A search for new phenomena in monojet events can be interpreted in terms of ADD models of large extra dimensions [1]. This interpretation is not discussed here, the reader is referred to [2].

From astronomical observations of its gravitational interactions, the presence of a non-baryonic dark matter (DM) component in the universe is derived ([3] for a review). While its nature is unknown, a weakly interacting massive particle (WIMP) is amongst the best candidates to make up DM. The fact that no SM particle has suitable properties suggests a new particle with a mass probably between a few $\mathrm{GeV}$ and $\mathrm{TeV}$. Such a particle might be pair-produced at the Large Hadron Collider (LHC) via some weak interaction, being either the known weak force or a new type of interaction. Since they would not interact in the detector, they could only be detected if initial- or final-state radiation (ISR/FSR) occurs, leading to signatures with e. g. one jet and large $E_{T}^{\text {miss }}$.

A model-independent description of DM pair production [5] in association with a jet is used to interpret the experimental limits measured in the analysis of monojet events. It assumes that the interaction between DM and SM particles is mediated by a much heavier particle, allowing to describe it within an effective field theory approach.

The detailed description of the analysis can be found in [2].

\section{Physics object reconstruction and analysis strategy}

For this analysis, jets are reconstructed from clusters of energy deposits in calorimeter cells, for which the anti- $k_{t}$ jet algorithm with a radius parameter of 0.4 is used [6]. The missing transverse momentum $\left(E_{T}^{\text {miss }}\right)$ is measured from energy deposits in the calorimeter up to $|\eta|$ of $4.5^{1}$ [7]. For the background estimate of $Z \rightarrow v v$, the $E_{T}^{\text {miss }}$ is defined disregarding the leptonic contribution to it in order to be able to compare the variables directly between regions in which the sensitivity to new physics signals is high (signal regions (SRs)) and those that are background-enriched and used for background estimation (conrtol regions (CRs)).

Electron candidates are restricted to have $p_{T}>20 \mathrm{GeV}$ and $|\eta|<2.47$ in order to be within the acceptance of the tracking system. Where for the electron veto in the signal regions (SRs) medium selection criteria for tracking and shower shape variables are applied (see [8] for details on the selection criteria) on electron candidates, the control region (CR) electrons for $W \rightarrow l v$ are required to be isolated and to fulfill tight selection criteria.

\footnotetext{
${ }^{1}$ ATLAS uses a right-handed coordinate system, originating in the centre of the detector. The z-axis lies along the beam direction, the $\mathrm{x}$-axis points to the centre of the LHC ring, and the $\mathrm{y}$-axis points upward. Polar coordinates $(\mathrm{r}, \varphi)$ are used in the transverse (x,y)-plane, $\varphi$ being the azimuthal angle around the beam pipe. The pseudorapidity $\eta$ is defined in terms of the polar angle $\theta$ as $\theta=\ln \tan (\theta / 2)$.
} 
A muon candidate is reconstructed either by associating a stand-alone muon spectrometer track with an inner detector track, or from an inner detector track that is confirmed by a directional segment in the muon spectrometer [9] (only for SR muons). Muons, which are used for the lepton veto applied in the SRs, are required to have $p_{T}>7 \mathrm{GeV}$ and $|\eta|<2.5$, where CR muons are restricted to $p_{T}>20 \mathrm{GeV}$ and $|\eta|<2.4$. They are also required to be isolated, where again the constraints for the CR muons are more stringent. To reject cosmic- muons a cut on the distance to the reconstructed primary vertex in $\mathrm{z}$ direction is also applied: $|z 0|<10 \mathrm{~mm}$.

\section{Event selection}

The events are selected according to criteria that achieve an enhancement of possible signal events in the sample. All events used for this analysis have to fulfill detector quality requirements and need to pass an inclusive trigger on $E_{T}^{\text {miss }}$. Further, the following selection criteria are applied:

- To reject non-collision background, a reconstructed vertex with at least 2 associated tracks with $p_{T}>400 \mathrm{MeV}$ is required.

- Events containing jets with $p_{T}>20 \mathrm{GeV}$ that do not pass standard quality criteria or that show anomalous charge or electromagnetic fraction or timing are rejected.

- The leading jet is required to have a charge fraction $<0.02$, to lie within $|\eta|<2$ and to have at least a transverse momentum of $120 \mathrm{GeV}$.

- Further, not more than two jets with $p_{T}>30 \mathrm{GeV}$ and $|\eta|<4.5$ are allowed.

- To reject back-to-back di-jet events, in which $E_{T}^{\text {miss }}$ comes from a faulty reconstruction of one of the jets, a cut on the angular separation of the second-leading jet and the $E_{T}^{\text {miss }}$ is applied: $\left|\Delta \phi\left(E_{T}^{\text {miss }}, p_{T}^{\text {jet } 2}\right)\right|>0.5$.

- A lepton veto on electrons and muons passing the SR selection described above is applied in the SRs to discriminate between electroweak (EW) backgrounds and possible signal events.

- In the CRs, the lepton veto is not applied, but the CR definition of muons and electrons is used to select $\mathrm{W}$ and $\mathrm{Z}$ events. The details are listed in Section 5.1.

From the data sample four inclusive signal regions (SR) are then constructed, according to the $E_{T}^{m i s s}$ and the $p_{T}$ of the leading jet. The thresholds are: $120 \mathrm{GeV}, 220 \mathrm{GeV}, 350 \mathrm{GeV}, 500 \mathrm{GeV}$. The $E_{T}^{m i s s}$ trigger is $98 \%$ efficient for the lowest SR (SR1) and more than $99 \%$ efficient for the other SRs.

\section{Data samples and simulations}

The ATLAS detector [10] at the LHC consists of an inner tracking detector, enclosed by a superconducting solenoid and electromagnetic and hadronic calorimeters, as well as by a muon spectrometer with integrated superconducting toroidal magnets. It covers almost the full solid angle around the collision point. The collision data is processed by a trigger system, organized 


\begin{tabular}{|c|ccc|}
\hline & Initial state & Type & Operator \\
\hline D1 & qq & scalar & $\frac{m_{q}}{M_{*}^{3}} \bar{\chi} \chi \bar{q} q$ \\
D5 & qq & vector & $\frac{1}{M_{*}^{2}} \bar{\chi} \gamma^{\mu} \chi \bar{q} \gamma_{\mu} q$ \\
D8 & qq & axial-vector & $\frac{1}{M_{*}^{2}} \bar{\chi} \gamma^{\mu} \gamma^{5} \chi \bar{q} \gamma_{\mu} \gamma^{5} q$ \\
D9 & qq & tensor & $\overline{M_{*}^{2}} \bar{\chi} \sigma^{\mu v} \chi \bar{q} \sigma_{\mu \nu} q$ \\
D11 & gg & scalar & $\frac{1}{4 M_{*}^{3}} \bar{\chi} \chi \alpha_{s}\left(G_{\mu \nu}^{a}\right)^{2}$ \\
\hline
\end{tabular}

Table 1: Effective interaction operators of WIMP pair production for which signal samples were simulated to be used in the limit-setting procedure.

in three layers, to decide if an event is recorded for further analysis. The presented analysis was performed on a time-integrated Luminosity of $4.7 \mathrm{fb}^{-1}$ with an uncertainty of 3.9\% [11].

In order to estimate the background remaining in the SRs Monte Carlo (MC) samples of EW background processes were generated with ALPGEN [12] and the parton distribution function (PDF) set CTEQ6L1 [13]. For all samples, the description of the parton shower and hadronization was provided by HERWIG [14], the underlying event by JIMMY [15]. The processes were also simulated using SHERPA [16], by comparison of these two generators an uncertainty on the modeling could be estimated. Further backgrounds are single top and top pair production, which are generated with MC@NLO [17] and the PDF set CTEQ6.6 [18]. Di-boson production was simulated with SHERPA.

The effective field theory approach for WIMP pair production [27] has an implementation in MADGRAPH5 [19], which was used to simulate WIMP pair production in association with one or two jets. WIMP masses between 10 and $1300 \mathrm{GeV}$ are simulated for four different interaction operators (D1, D5, D9, D11, see Table 1). A lower cut on the transverse momentum of $80 \mathrm{GeV}$ was imposed on the leading parton. The simulation only regards the four lightest quarks, assuming equal coupling to the DM particles. The generated events are then interfaced to PYTHIA [20] for the addition of parton shower, hadronization and underlying event, using CTEQ6L1 as PDF. In order to match the matrix-element calculations of MADGRAPH to the parton shower simulation of PYTHIA, the MLM matching scheme is used [21]. The factorization and renormalization scales are defined by: $\sum \sqrt{m^{2}+p_{T}^{2}}$, where the sum goes over all particles produced.

The interaction of the particles with the detector was simulated with GEANT4 [22] in case of the background MC samples and by using a less CPU-consuming detector simulation based on parametrisation of calorimeter responses, ATLFASTII [23], for signal samples.

\section{Background estimation}

The most important background is the production of a $\mathrm{Z}$ boson that decays into two neutrinos, leading to $E_{T}^{\text {miss }}$. This background is irreducible, whereas other backgrounds can be in principle distinguished from signal events. They might nevertheless pass the selection due to a lepton being outside of the acceptance or missed because of finite identification efficiencies, or a mis-measurement of jet $p_{T} / E_{T}^{\text {miss }}$. All EW backgrounds are determined by a data-driven estimate, where the yield in control regions is transferred to signal region yields. A small multijet background, coming from QCD events where for one ore more jets the momentum is mis-measured, 
is also estimated from data, as well as the non-collision-background, whereas top and di-boson backgrounds are determined solely from MC.

\subsection{Electroweak backgrounds}

For the estimation of the EW backgrounds control regions are defined by explicitly choosing events in which $\mathrm{W}$ or $\mathrm{Z}$ bosons are produced and decay leptonically. The jet $p_{T}$ and $E_{T}^{\text {miss }}$ requirements are kept identically to the SR selection to mimic the SRs as close as possible. The shape of observables like $E_{T}^{m i s s}$ and jet $p_{T}$ as well as the efficiency related to the lepton identification is estimated from data. For the transfer from SR to CR only ratios of MC quantities enter, which results in reduced systematic uncertainties since many of them cancel in such ratios.

The detailed construction of the four control regions is defined below:

- $W \rightarrow e v+$ jets: events are required to pass an electron trigger $\left(p_{T}>20\right.$ or $\left.22 \mathrm{GeV}\right)$. For the electron definition, the tighter $\mathrm{CR}$ selection, described above, is used. Exactly one electron is required, fulfilling $p_{T}>25 \mathrm{GeV}$. Further electrons or muons are vetoed. If $\Delta R(e, j e t)<0.2$, the jet is not considered as a jet anymore but as corresponding to the electron. A cut on $E_{T}^{\text {miss }}>25 \mathrm{GeV}$ and $40<m_{T}<100 \mathrm{GeV}$ for the transverse mass $m_{T}=$ $\sqrt{2 p_{T}^{\ell} E_{T}^{\text {miss }}\left(1-\cos \Delta \phi\left(p_{T}^{\ell}, E_{T}^{\text {miss }}\right)\right)}$, using the lepton $p_{T}^{\ell}$, improves the purity of $\mathrm{W}$ events. The requirements for $E_{T}^{\text {miss }}$ and jet $p_{T}$ match those in the SRs, but if the $Z \rightarrow v v$ background is estimated, the $E_{T}^{\text {miss }}$ contribution from the electron is subtracted to mimic the SR $E_{T}^{\text {miss }}$ more closely.

- $W \rightarrow \mu v+$ jets: the SR trigger on $E_{T}^{\text {miss }}$ is applied. The muon candidates are selected according to the tighter CR criteria, exactly one muon is required, further electrons or muons are vetoed. Again, the purity of $\mathrm{W}$ events is enhanced by applying a cut on the transverse mass: $m_{T}>40 \mathrm{GeV}$ and on the $E_{T}^{\text {miss }}$ (including the muon contribution): $E_{T}^{\text {miss }}>25 \mathrm{GeV}$. For the further classification according to $E_{T}^{\text {miss }}$ the standard calorimeter-based $E_{T}^{\text {miss }}$ variable, which does not contain muon information, is used, as for the SRs.

- $Z \rightarrow e e+$ jets: as for $W \rightarrow e v+$ jets, electron triggers are used to select relevant events. Two electrons with opposite charges are required, passing the CR selection as well as $p_{T}>25$ $\mathrm{GeV}$ for the leading electron and $p_{T}>20 \mathrm{GeV}$ for the sub-leading one. The electrons are required to fulfill $66<m_{e^{+} e^{-}}<116 \mathrm{GeV}$ to enhance the purity of $\mathrm{Z}$ events. As above, events containing other electrons or muons are rejected and an overlap removal for jets and electrons is applied. For the estimation of the $Z \rightarrow v v$ background, the $E_{T}^{\text {miss }}$ excluding the electron contribution, is considered.

- $Z \rightarrow \mu \mu+$ jets: the SR $E_{T}^{\text {miss }}$ trigger selection is applied. Similar to the $Z \rightarrow e e+$ jets channel, two opposite-charged muons are required to fulfill the constraint on their invariant mass to enhance the fraction of $\mathrm{Z}$ events. Again, further electrons or muons are vetoed. The standard calorimeter-based $E_{T}^{\text {miss }}$ variable is used for the background estimation.

Having defined the above CRs, the remaining background in the SRs, $N_{S R}^{\text {predicted }}$ can be estimated. The event yield in the CR, $N_{C R}^{\text {data }}$ is corrected for small remaining background, $N_{C R}^{b k g}$. Then, 
it is transferred to the SR yield by the use of a transfer factor $T$ relating the number of simulated events in the CR to those in the SR, and by applying a correction factor $C$, inferred form simulation and measurement:

$$
\begin{aligned}
N_{S R}^{\text {predicted }}=\left(N_{C R}^{\text {Data }}\right. & \left.-N_{C R}^{\text {Bkg }}\right) \cdot C \cdot T \\
& =\left(N_{C R}^{\text {Data }}-N_{C R}^{\text {multijet }}\right) \cdot\left(1-f_{E W}\right) \times \frac{\varepsilon_{E_{T}^{\text {miss }}}^{\text {trig }} \cdot \mathcal{L}_{E_{T}^{\text {miss }}}}{A_{\ell} \cdot \varepsilon_{\ell} \cdot \varepsilon_{Z / W} \cdot \varepsilon_{\ell}^{\text {trig }} \cdot \mathcal{L}_{\ell}} \times \frac{N_{S R}^{M C}}{N_{j e t / E_{T}^{m i s s}}^{M C}} .
\end{aligned}
$$

The terms in the second line of Equation 5.1 refer to the following quantities:

- $N_{C R}^{\text {multijet }}$ denotes the QCD background, which is estimated by electron-fake enriched samples. For $W \rightarrow e v$ it is estimated to be $1-2 \%$, whereas its contribution to other control region is negligible.

- $f_{E W}$ refers to the fraction of events remaining from other EW or SM processes. Its values determined from simulation are $2 \%$ for $\mathrm{Z}$ boson decays into other flavors than the selected one and $10 \%$ for $\mathrm{W}$ boson decays respectively, whereas top and di-boson contributions are negligible. Note that $f_{E W}$ denotes a ratio of MC event yields and hence many systematics drop out.

- $A_{\ell}$ is the lepton acceptance, determined from simulation, $\varepsilon_{\ell}$ their identification efficiency estimated from data. $\varepsilon_{Z / W}$ is the efficiency of the criteria applied to select $\mathrm{Z}$ or $\mathrm{W}$ bosons. These contributions to the correction factor occur because the lepton and boson selection is only applied in the CRs and not in the SR selection.

- Since for electron CRs an electron trigger and not the $E_{T}^{\text {miss }}$ trigger applied in the SRs is required, the ratio of electron trigger efficiency, $\varepsilon_{\ell}^{\text {trig }}$, times its corresponding luminosity, $\mathcal{L}_{\ell}$, to the same quantities for the $E_{T}^{\text {miss }}$ trigger, $\varepsilon_{E_{T}^{\text {miss }}}^{\text {trig }}$ and $\mathcal{L}_{E_{T}^{\text {miss }}}$, enter the correction for electron CRs.

- The transfer factor $T$ contains the number of simulated events for the process that should be estimated in the SR, $N_{S R}^{M C}$, and the number of simulated events for the CR, with the effect of the lepton efficiency and acceptance already taken out and regarding the $E_{T}^{\text {miss }}$ without leptons. Again, only a ratio of simulated quantities enter.

The number of background events is estimated in bins of the variable in question. For the final results, bins in $E_{T}^{\text {miss }}$ were taken. All the different estimates for $Z \rightarrow v v$ are combined in the end to obtain the final number.

The background estimate was cross-checked by varying the CR definitions: first, an inclusive CR was constructed by just inverting the lepton veto of the SR selection, leading to a W/Z enriched sample. Second, the lepton definition in the CR selection was loosened in order to exactly invert the SR veto. In both cases, the results were found to be consistent with the standard estimation. 


\subsection{Multijet and non-collision background}

The multijet background resulting from events in which one or more jets are mis-reconstructed is not well modeled in simulations and hence has to be estimated from data by selecting a sample of events, required to pass all SR selection cuts but the jet veto or the angular cut on the direction of the $E_{T}^{\text {miss }}$ and the second-leading jet. Top- or Z/W backgrounds in these samples are estimated from $\mathrm{MC}$ and simulation scaled to data, respectively, and are subtracted.

A straight can be fitted to the $p_{T}$ distribution of the second- or third-leading jet in order to estimate the number of jets that fall below the threshold of $p_{T}=30 \mathrm{GeV}$ and are missed by the selection criteria. This background amounts to at most 1\% in SR 1, 2 and 3 and is negligible when going to even higher $E_{T}^{m i s s}$ and jet $p_{T}$ in SR 4.

As the multijet background, the non-collision background contributes most for lower SRs, namely less than 1\% in SR1 and 2. It is estimated from a data sample requiring a beam-background muon going along the beam direction in the event. About 20-50\% of non-collision events can be tagged like that, which was estimated from data of unpaired proton-bunches. More details can be found in [24].

\subsection{Systematic Uncertainties}

The dominant systematic uncertainties arise from the simuation of the kinematic distributions of the background, since their shape affect the transfer factor, together with the experimental uncertainties on JES and $E_{T}^{m i s s}$. Further sources of uncertainties are coming from the finite resolution and overall scale of the muon momentum, the estimated lepton reconstruction and trigger efficiencies as well as from the limited statistics of the MC samples used for determining the transfer factor, the estimation of $f_{E W}$ and the multijet background estimate. Negligible are the uncertainties related to pile-up, electron energy measurement and JER.

The multijet background is estimated to have an uncertainty of $100 \%$, for the non-collision background it is $10 \%$. The JES and JER uncertainties are assessed by combining data-driven and simulation-based approaches, which is described in [6] in more detail. These uncertainties are propagated to obtain the uncertainty on $E_{T}^{\text {miss }}$.

The comparison of different generators allows to rate the uncertainties on the shape of kinematic distributions and cross-sections in $\mathrm{Z}$ or $\mathrm{W}$ events as well as on $f_{E W}$. Used were ALPGEN/SHERPA and ALPGEN/PYTHIA, respectively. For the MC-based estimate on top and diboson backgrounds an uncertainty of $20 \%$ is assumed, dominated by the JES uncertainty.

\section{Results}

In all four regions the predictions from SM background processes agree well with the measured number of events within the statistical and systematic uncertainties. Figure 1 compares the measured distributions of $E_{T}^{\text {miss }}$, leading jet $p_{T}$ and sub-leading jet $p_{T}$ with the SM expectation (histogram) in SR1 (left) and SR4 (right). The shapes as well as the normalizations agree well in all cases. To illustrate the impact from signals BSM, simulated ADD and WIMP signals are included in the plots as dashed lines.

The fact that no excess above the SM is observed is then translated into $90 \%$ and $95 \%$ confidence limits (CL) on the visible cross section, taking into account the acceptance and efficiency 

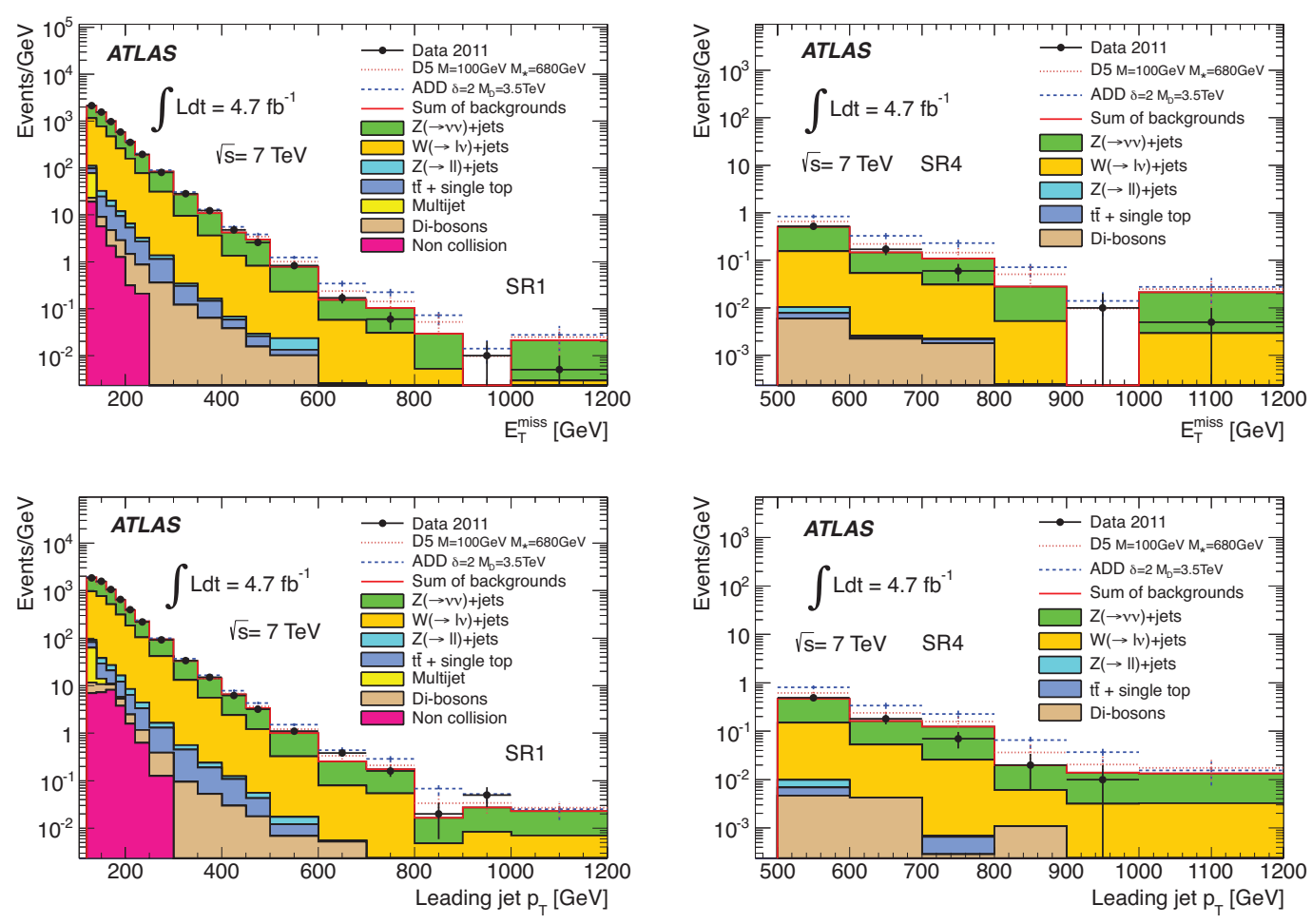

Figure 1: Distributions of $E_{T}^{\text {miss }}$ and leading jet $p_{T}$ for signal regions SR1 on the left and SR4 on the right. ADD and WIMP signals for cross sections equal to the excluded values are drawn as dashed lines on top of the predicted background distributions (histogram).

of the analysis: $\sigma \times A \times \varepsilon$ (details on the limit setting procedure can be found in [25]). For this purpose, event yields are integrated over the signal region.

The resulting visible cross-section limits on any BSM model showing up in monojet events are summarized in Table 2. Typical efficiencies of selection criteria related to jets and $E_{T}^{\text {miss }}$ of $\varepsilon \sim 83 \%$ are found for signal and EW background samples.

\section{Interpretation: WIMP pair production}

If the mass of WIMPs allows them to be pair-produced at the LHC, these particles would escape the detector unseen. Only if initial- or final-state radiation is giving a jet, a photon or a boson that balances the WIMP pair such a process can be detect via the large $E_{T}^{\text {miss }}$ in the event coming from the undetected WIMP pair.

The model-independent approach used to interpret the measurement needs to make some general assumptions, although it does not rely on details of any model beyond the SM (BSM). The approach assumes that only the WIMP candidates can be produced at the LHC, all other potential new particles, also the ones mediating the interaction between WIMPs and the SM particles, are assumed to be out of the LHC reach. Hence, an effective field theory approach can be applied and the mediating particle can be integrated out, leading to the picture of a contact interaction between WIMPs and SM particles. For the presented results the DM particle is assumed to be a Dirac 


\begin{tabular}{|c|cccc|}
\hline & SR1 & SR2 & SR3 & SR4 \\
\hline$Z \rightarrow v v+$ jets & $63000 \pm 2100$ & $5300 \pm 280$ & $500 \pm 40$ & $58 \pm 9$ \\
$W \rightarrow \tau \nu+j e t s$ & $31400 \pm 1000$ & $1853 \pm 81$ & $133 \pm 13$ & $13 \pm 3$ \\
$W \rightarrow e v+$ jets & $14600 \pm 500$ & $679 \pm 43$ & $40 \pm 8$ & $5 \pm 2$ \\
$W \rightarrow \mu v+$ jets & $11100 \pm 600$ & $704 \pm 60$ & $55 \pm 6$ & $6 \pm 1$ \\
$t \bar{t}$, single $t$ & $1240 \pm 250$ & $57 \pm 12$ & $4 \pm 1$ & - \\
Multijets & $1100 \pm 900$ & $64 \pm 64$ & $8_{-8}^{+9}$ & - \\
Non-coll. Background & $575 \pm 83$ & $25 \pm 13$ & - & - \\
$Z / \gamma^{*} \rightarrow \tau \tau+$ jets & $421 \pm 25$ & $15 \pm 2$ & $2 \pm 1$ & - \\
Di-Boson & $302 \pm 61$ & $29 \pm 5$ & $5 \pm 1$ & $1 \pm 1$ \\
$Z / \gamma^{*} \rightarrow \mu \mu+j e t s$ & $204 \pm 19$ & $8 \pm 4$ & - & - \\
\hline Total Background & $124000 \pm 4000$ & $8800 \pm 400$ & $750 \pm 60$ & $83 \pm 14$ \\
Measured Events & 124703 & 8631 & 785 & 77 \\
\hline$\sigma_{v i s}^{\text {obs }}$ at $90 \%[\mathrm{pb}]$ & 1.63 & 0.13 & 0.026 & 0.005 \\
$\sigma_{v i s}^{\text {exp }}$ at $90 \%[\mathrm{pb}]$ & 1.54 & 0.15 & 0.020 & 0.0064 \\
$\sigma_{v i s}^{\text {obs }}$ at $95 \%[\mathrm{pb}]$ & 1.92 & 0.17 & 0.030 & 0.0069 \\
$\sigma_{v i s}^{\text {exp }}$ at $95 \%[\mathrm{pb}]$ & 1.82 & 0.18 & 0.024 & 0.0079 \\
\hline
\end{tabular}

Table 2: Shown are the estimated backgrounds in the four SR, as well as the measured number of events in data. Observed and expected limit on the visible cross section of any BSM contribution are computed at $90 \%$ and $95 \% \mathrm{CL}$.

fermion, but conclusions for Majorana fermions can also be drawn since the cross section in this case is proportional to the one for Dirac fermions and only differs by a factor of two. With these assumptions, the interaction between SM and DM particles are defined by only two parameters, namely the mass of the DM particle, $m_{\chi}$, and the suppression scale $M_{*}$, which is related to the mediator mass as well as to its couplings to SM and DM particles.

In total, 14 interaction operators can be constructed for Dirac fermions (for Majorana fermions the ones corresponding to vector interactions are not allowed). They fall into four classes regarding the resulting $E_{T}^{\text {miss }}$ and jet spectra. Hence, a characteristic set of one operator per class was chosen here for limit-setting (see Table 1): D1, D5, D9 and D11. Limits for D8, which falls into the same class as D5, were also calculated since it is often used for comparisons. The acceptance of D5 was assumed, whereas the proper D8 cross sections were used. Where D1, D5, D8 and D9 describe $q q \rightarrow \chi \chi$ processes, D11 refers to gluons in the initial state: $g g \rightarrow \chi \chi$.

In general, the effective field theory approach is considered conservative: also in regimes where the validity might be questioned the cross section is mostly underestimated, compared to the full theory, which leads to more conservative limits. A detailed discussion can be found in [27]. Having this in mind, the effective theory provides a useful framework for comparing LHC results to direct or indirect dark matter searches.

Experimental and theoretical systematic uncertainties are considered when setting limits on the model parameters $M_{*}$ and $m_{\chi}$. The experimental uncertainties on JES, JER, and $E_{T}^{m i s s}$ are taken to be fully correlated with the ones determined for the background estimate and range from 1$20 \%$ uncertainties on the WIMP event yield, depending on the signal region and the considered 
interaction operator. Further, experimental uncertainties associated with the trigger efficiency (1\%) and the luminosity measurement (3.9\%) affect the WIMP event yield.

Theoretical uncertainties on the simulated WIMP signal result from the PDF set used, the amount of ISR/FSR that was assumed, and the factorization and renormalization scales set in the signal simulation. The PDF uncertainties are determined from CTEQ6M error sets. The ISR/FSR uncertainties are estimated by varying the jet matching scale between MADGRAPH5 and PYTHIA within twice and half the nominal value and by changing the QCD scale $\alpha_{s}$ of PYTHIA within a range that is consistent with experimental data [26]. Depending on the signal region, the resulting uncertainties on $\sigma \times A$, added in quadrature, range from 3-5\% for the matching scale and 4-6\% for $\alpha_{s}$. The dependence of the ISR/FSR uncertainties on the choice of operator is found to be negligible. PDF uncertainties mostly affect the signal cross sections, the acceptance do not change significantly. They are found to depend on the choice of operator and not the particular signal region since overall cross-section differences affect all SRs in a similar way. Uncertainties ranging from $4 \%$ and 5\% for operators D9 and D5 to $16 \%$ and $18 \%$ for D11 and D1 are found. The dominating theoretical systematic uncertainty results from the factorization and renormalization scales: varying these scales by factors of two or a half results in a $30 \%$ signal uncertainty, independent of the considered operator or the signal region.

From the limit on the visible cross section of new physics processes BSM, lower limits on the suppression scale $M_{*}$ as a function of the WIMP mass $m_{\chi}$ can be derived. Shown in Figure 2 are the $90 \%$ CL lower limits for all considered operators, where the SR with the best expected limits was chosen. The limits are calculated from simulated samples produced for $m_{\chi}$ between 10 and $1300 \mathrm{GeV}$. Since no significant change in cross section or acceptance is found when going to lower WIMP masses, they can be extrapolated down to $m_{\chi}=1 \mathrm{GeV}$, until the regime of warm or hot DM would start. The observed limit on $M_{*}$ includes experimental uncertainties, the effect of theoretical uncertainties is indicated by dotted red $\pm 1 \sigma$ lines above and below it. Around the expected limit, $\pm 1 \sigma$ variations due to statistical and systematic uncertainties are shown as a gray band.

For all operators, the lower limits are flat up to $m_{\chi}=100 \mathrm{GeV}$ and get worse around $m_{\chi}=200$ $\mathrm{GeV}$ due to the limited collision energy. Note that the $M_{*}$ limits for D1 are quantitatively much smaller due to the inclusion of a factor $m_{q} / M_{*}$ in the definition of the operator (see Table 1). In the bottom-right corner of the $m_{\chi}-M_{*}$ plane the effective field theory approach is no longer valid, which is indicated by the light-gray shaded area. Only small parts of the limit curves on $M_{*}$ fall into that region. In any case, such a region of validity cannot be precisely defined without detailed knowledge of the underlying physics model BSM. Also included are lines corresponding to the coupling that would lead to the measured thermal relic density (taken from [27]) in the absence of any other interaction than the one considered.

Within the effective field theory approach, the bounds on $M_{*}$ in dependence on the DM mass $m_{\chi}$ can be translated into limits on cross sections of WIMP-nucleon scattering [27]. In this way, the ATLAS collider bounds can be compared to the results of direct DM experiments, looking for scattering of WIMPs off nucleons at very low momentum transfer. Depending on the considered interaction operator spin-dependent (Figure 3) or spin-independent (Figure 3) contributions can be calculated. The spin-dependent limits, derived from the operators D8 and D9, give a smaller, hence better, bound on the WIMP-nucleon cross section throughout the range of $m_{\chi}$, compared to direct DM experiments. In the spin-independent case the bounds from direct detection experiments are 

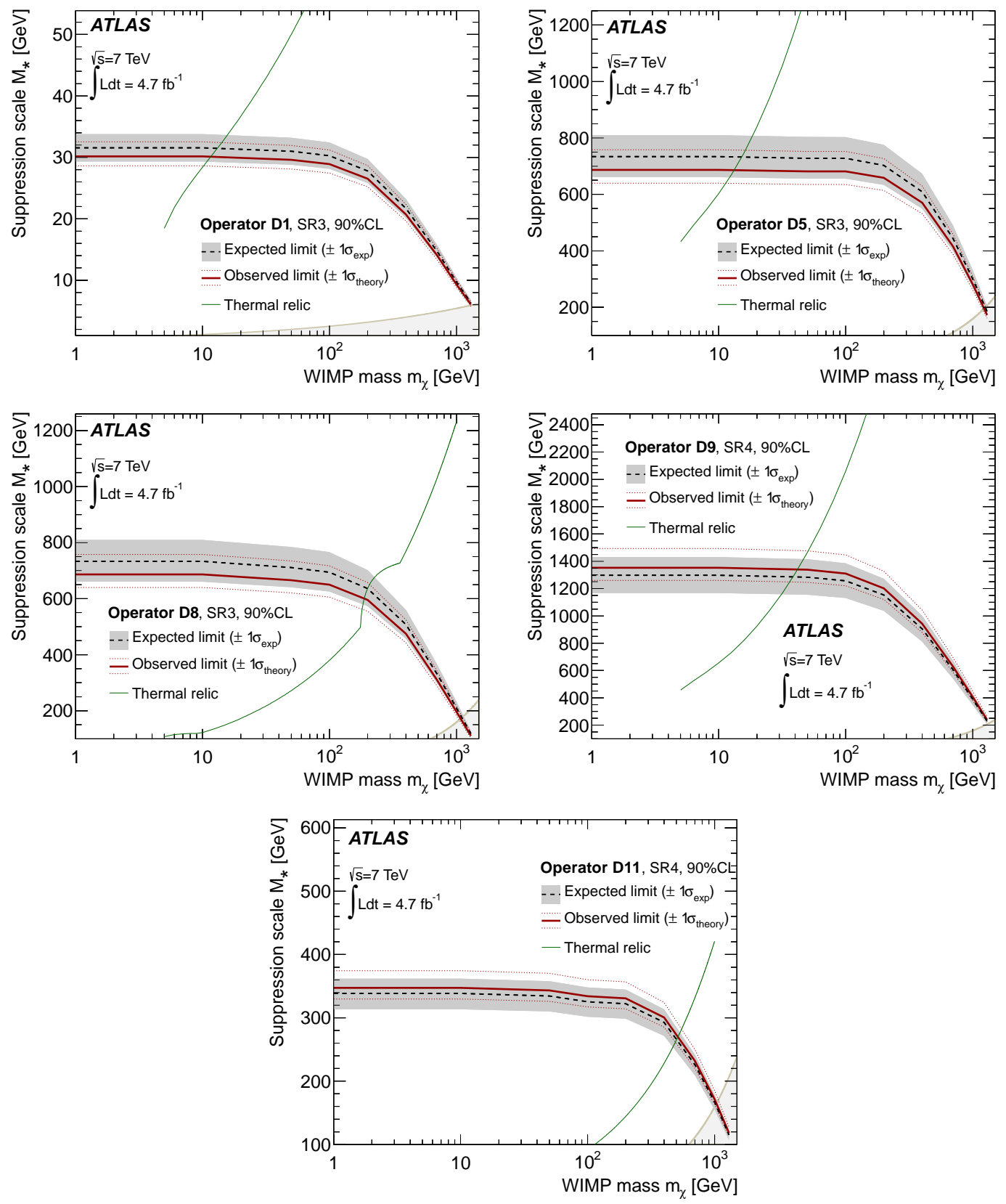

Figure 2: Lower limits at 90\% CL on $M_{*}$ for different masses of the DM particle $\chi$. Observed and expected limits are shown as dashed black and red solid lines, respectively. The gray $\pm 1 \sigma$ band around the expected limit is the variation within statistical and systematic uncertainties on SM and signal processes. The observed limit includes all but the theoretical signal uncertainties, which are indicated by the red dotted $\pm 1 \sigma$ limit lines around it. The $M_{*}$ values for which WIMPs of the given mass could be consistent with the measured relic abundance [27] are shown as green lines, assuming annihilation in the early universe happened exclusively via the given operator. The shaded light-gray regions in the bottom right corners indicate where the effective field theory approach breaks down [27]. The plots for D1, D5, D8 are based on SR3, the ones for D9 and D11 rely on SR4. 

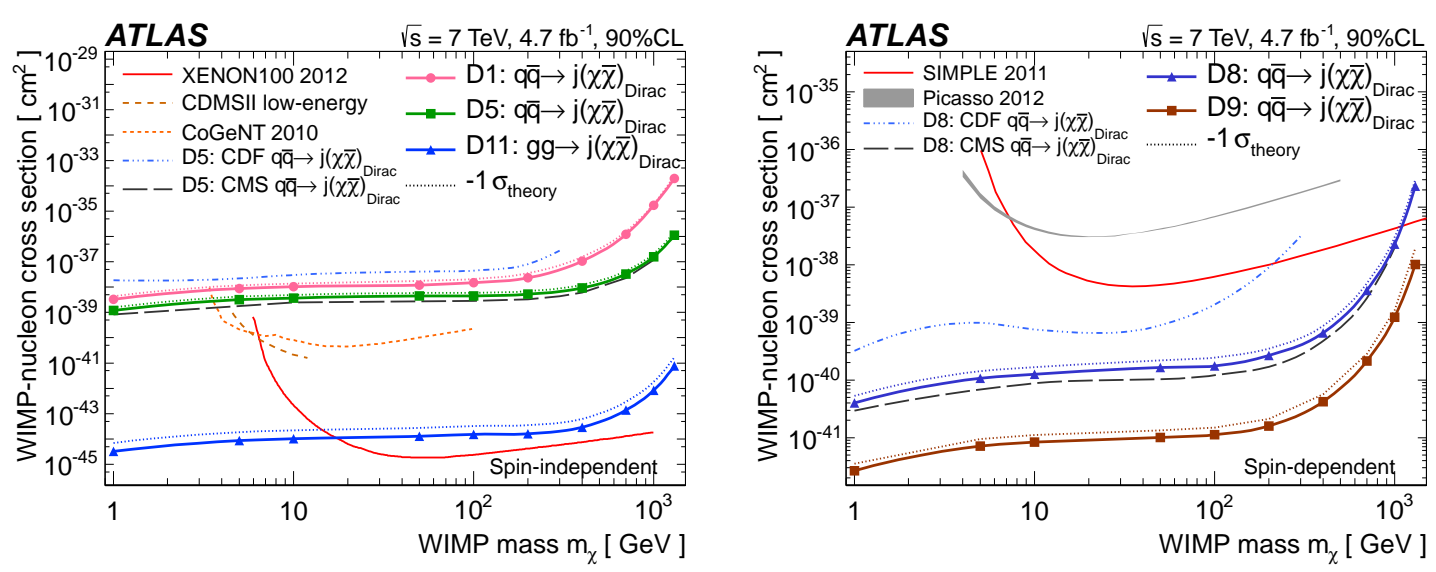

Figure 3: Inferred 90\% CL ATLAS limits on spin-independent (left) and spin-dependent (right) WIMPnucleon scattering. Cross section bounds are shown versus WIMP mass $m_{\chi}$. The solid lines represent the observed limits excluding theoretical uncertainties, the dotted lines indicate theoretical uncertainties. The spin-independent limits are compared to 90\% CL limits from the XENON100 [28], CDMSII [29], CoGeNT [30], CDF [31], and CMS [32] experiment. The spin-dependent limits are compared to 90\% CL limits from the SIMPLE [33], Picasso [34], CDF [31], and CMS [32] experiment.

stronger for $m_{\chi}$ of about $10 \mathrm{GeV}$ and larger, whereas the collider bounds get important for the region of low DM masses. Especially powerful is the bound in case of the gluon operator D11, it is competitive to direct detection bounds up to $m_{\chi}$ of $20 \mathrm{GeV}$.

The collider limits on vector and axial-vector interactions are also interpreted in terms of the relic abundance of WIMPs, using the same effective theory approach [27]. The upper limits on the annihilation rate of WIMPs into light quarks are shown in Figure 4. The annihilation rate is defined as the product of the annihilation cross section $\sigma$ and the relative WIMP velocity $v$, averaged over the WIMP velocity distribution: $\langle\sigma v\rangle$. The results are compared to limits on WIMP annihilation to $b \bar{b}$, obtained from galactic high-energy gamma-ray observations, measured by the Fermi-LAT experiment [35]. Gamma-ray spectra from WIMPs annihilating to $b \bar{b}$ are taken to be comparable to those from annihilation to lighter quarks [36]. Under this assumption, the ATLAS and FermiLAT limits can be compared, noting that the Fermi-LAT values correspond to Majorana fermions and are hence scaled up by a factor of two. Again, the ATLAS bounds are especially important for small WIMP masses, below $10 \mathrm{GeV}$ for vector couplings and below about $100 \mathrm{GeV}$ for axial-vector couplings. In this region, the ATLAS limits are below the annihilation cross section needed to be consistent with the thermic relic value, still under the assumption that WIMPs have annihilated to SM quarks only via the particular operator in question. For masses of $m_{\chi} \geq 200 \mathrm{GeV}$ the ATLAS sensitivity becomes worse than the one of Fermi-LAT. In this region, improvements can be expected when going to larger center-of-mass energies at the LHC.

The effective field theory approach describes all WIMP-SM particle interaction by only two parameters, $M_{*}$ and $m_{\chi}$, and hence allows to connect WIMP pair production at the LHC, WIMPnucleon scattering measured by direct-detection experiments and WIMP annihilation measured by indirect-detection experiments. The approach relies on the assumption of validity of the effective field theory approach and the presented comparisons are restricted to interactions via one single op- 


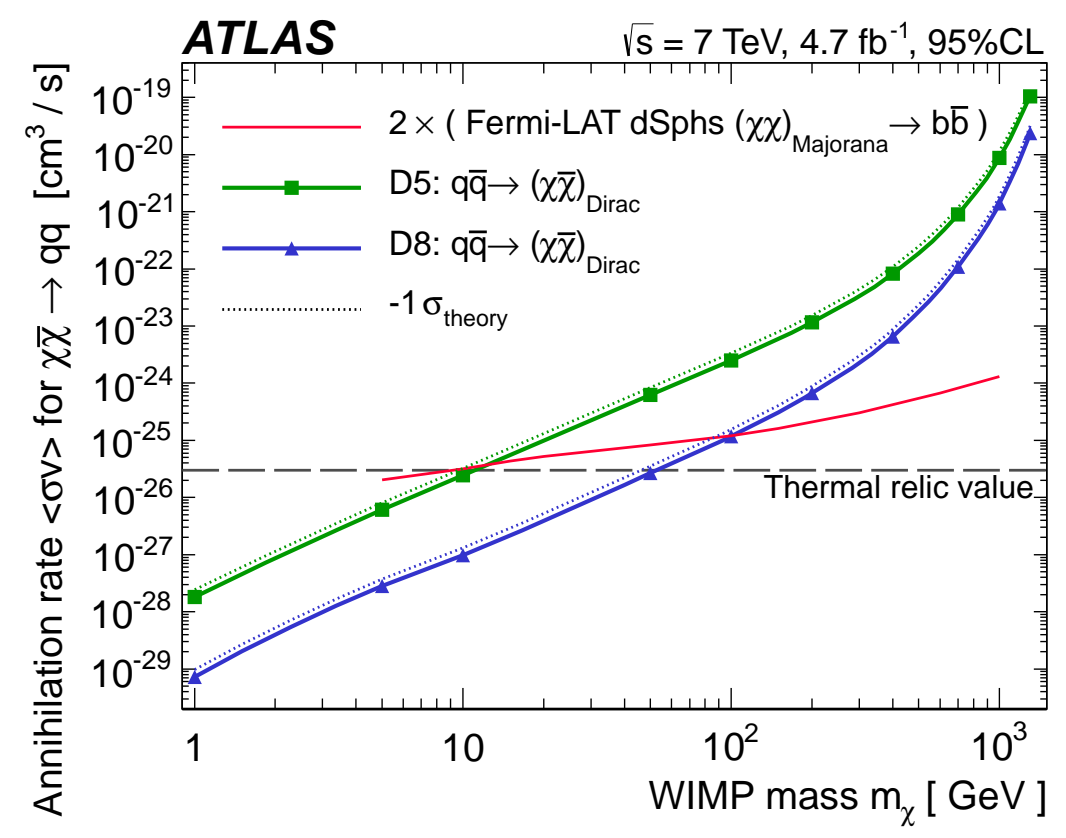

Figure 4: ATLAS 95\% CL limits on WIMP annihilation rates $\langle\sigma v\rangle$ in dependence on the dark matter mass $m_{\chi} .\langle\sigma v\rangle$ is calculated according to [27]. The solid lines represent the observed limits excluding theoretical uncertainties, the dotted lines indicate theoretical uncertainties. For comparison, high-energy gamma-ray limits from observations of Galactic satellite galaxies with the Fermi-LAT sattelite [35] for Majorana WIMPs are shown. The Fermi-LAT limits are scaled up by a factor of two to make them comparable to the ATLAS Dirac WIMP limits. The horizontal line indicates the value required for WIMPs to make up the relic abundance set by the WMAP measurement [4].

erator at a time, since mixing or interference effects are not considered. Nevertheless, the approach allows a valuable comparison of complementary results on DM detection: if any of the experiments sees a signal, the interpretation in this approach can lead to further insights on the nature of DM as well as the underlying physics by a comparison of different techniques and observables.

\section{Summary}

A search for new phenomena in monojet events is presented for $4.7 \mathrm{fb}^{-1}$ of data recorded with the ATLAS experiment at the LHC in proton-proton collisions at $\sqrt{s}=7 \mathrm{TeV}$. The dominant Standard Model backgrounds from $\mathrm{Z}$ and $\mathrm{W}$ events in association with jet(s), where the boson decays to a final state that includes neutrinos and hence $E_{T}^{\text {miss }}$, are determined using data control regions with correction and transfer factors determined from data and simulations. This technique allows a precise estimate of the SM contributions to monojet final states with a small total uncertainty of $3.2 \%$ for the background prediction in the high statistics signal region SR1. In each of the four signal regions, defined according to the $E_{T}^{\text {miss }}$ and leading jet $p_{T}(120,220,350$ and $500 \mathrm{GeV})$, agreement is found between the Standard Model predictions and the data. Upper limits are set at 90\% and $95 \%$ CL on the visible cross section of any additional contribution to the signal regions. These limits $(95 \% \mathrm{CL}$ ) range from $1.92 \mathrm{pb}$ in the first signal region to $7 \mathrm{fb}$ in the fourth signal 
region. The cross-section upper limits are interpreted within an effective field theory, giving limits on the suppression scale $M_{*}$ for pair production of dark matter particles. Within this approach, the ATLAS limits can be converted to limits on WIMP-nucleon scattering and WIMP annihilation cross sections. Assuming the effective field theory is valid and the WIMPs can be pair-produced at the LHC, the limits are complementary and competitive to limits set by direct and indirect dark matter detection experiments, in particular at small WIMP masses of $m_{\chi}<10 \mathrm{GeV}$.

\section{References}

[1] N. Arkani-Hamed, S. Dimopoulos, and G. Dvali, Phys.Lett. B429 (1998) 263-272, arXiv:hep-ph/9803315 [hep-ph].

[2] ATLAS Collaboration, arXiv:1210.4491v1 [hep-ex].

[3] G. Bertone, D. Hooper, and J. Silk, Phys.Rept. 405 (2005) 279-390, arXiv:hep-ph/0404175 [hep-ph].

[4] WMAP Collaboration, Astrophys.J.Suppl. 192 (2011) 18, arXiv:1001.4538 [astro-ph.CO].

[5] M. Beltran, D. Hooper, E. W. Kolb, Z. A. Krusberg, and T. M. Tait, JHEP 1009 (2010) 037, arXiv:1002.4137 [hep-ph].

[6] ATLAS Collaboration, Eur. Phys. J. C, 733 (2013) 2304

[7] ATLAS Collaboration, Eur.Phys.J. C72 (2012) 1844, arXiv:1108.5602 [hep-ex].

[8] ATLAS Collaboration, Eur.Phys.J. C72 (2012) 1909, arXiv:1110.3174 [hep-ex].

[9] ATLAS Collaboration, JHEP 1012 (2010) 060, arXiv:1010.2130 [hep-ex].

[10] ATLAS Collaboration, JINST 3 (2008) S08003.

[11] ATLAS Collaboration, Eur.Phys.J. C71 (2011) 1630, arXiv:1101.2185 [hep-ex].

[12] M. L. Mangano, M. Moretti, F. Piccinini, R. Pittau, and A. D. Polosa, JHEP 0307 (2003) 001, arXiv:hep-ph/0206293 [hep-ph].

[13] J. Pumplin, D. Stump, J. Huston, H. Lai, P. M. Nadolsky, et al., JHEP 0207 (2002) 012, arXiv:hep-ph/0201195 [hep-ph].

[14] G. Corcella, I. Knowles, G. Marchesini, S. Moretti, K. Odagiri, et al., arXiv:hep-ph/0210213 [hep-ph].

[15] J. Butterworth, J. R. Forshaw, and M. Seymour, Z.Phys. C72 (1996) 637-646, arXiv:hep-ph/9601371 [hep-ph].

[16] T. Gleisberg, S. Hoeche, F. Krauss, M. Schonherr, S. Schumann, et al., JHEP 0902 (2009) 007, arXiv:0811.4622 [hep-ph].

[17] S. Frixione and B. R. Webber, arXiv:hep-ph/0601192 [hep-ph].

[18] P. M. Nadolsky, H.-L. Lai, Q.-H. Cao, J. Huston, J. Pumplin, et al., Phys.Rev. D78 (2008) 013004, arXiv:0802.0007 [hep-ph].

[19] J. Alwall, M. Herquet, F. Maltoni, O. Mattelaer, and T. Stelzer, JHEP 1106 (2011) 128, arXiv:1106.0522 [hep-ph].

[20] T. Sjostrand, S. Mrenna, and P. Z. Skands, JHEP 0605 (2006) 026, arXiv:hep-ph/0603175 [hep-ph].

[21] M. L. Mangano, M. Moretti, F. Piccinini, and M. Treccani, JHEP 0701 (2007) 013, arXiv:hep-ph/0611129 [hep-ph]. 
[22] GEANT4 Collaboration, S. Agostinelli et al., Nucl. Instrum. Meth. A506 (2003) 250âĂŞ303.

[23] ATLAS Collaboration, Eur. Phys. J. C70 (2010) 823âĂŞ874, arXiv:1005.4568 [physics.ins-det].

[24] ATLAS Collaboration, arXiv:1303.0223 [hep-ex]

[25] G. Cowan, K. Cranmer, E. Gross, and O. Vitells, Eur.Phys.J. C71 (2011) 1554, arXiv:1007.1727 [physics.data-an].

[26] P. Z. Skands, Phys.Rev. D82 (2010) 074018, arXiv:1005.3457 [hep-ph].

[27] J. Goodman, M. Ibe, A. Rajaraman, W. Shepherd, T. M. Tait, et al., Phys.Rev. D82 (2010) 116010, arXiv:1008.1783 [hep-ph].

[28] XENON100 Collaboration, E. Aprile et al., arXiv:1207.5988 [astro-ph.CO].

[29] CDMS Collaboration, Z. Ahmed et al., Phys. Rev. Lett. 106 (2011) 131302. http://link.aps.org/doi/10.1103/PhysRevLett.106.131302.

[30] CoGeNT Collaboration, C. Aalseth et al., Phys.Rev.Lett. 106 (2011) 131301, arXiv:1002.4703 [astro-ph.CO].

[31] CDF Collaboration, T. Aaltonen et al., Physical Review Letters 108 no. 21, (2012) 211804.

[32] CMS Collaboration, arXiv:1206.5663 [hep-ex].

[33] M. Felizardo, T. Girard, T. Morlat, A. Fernandes, A. Ramos, et al., Phys.Rev.Lett. 108 (2012) 201302, arXiv:1106.3014 [astro-ph.CO].

[34] PICASSO Collaboration, S. Archambault et al., Phys.Lett. B711 (2012) 153-161, arXiv:1202.1240 [hep-ex].

[35] Fermi-LAT Collaboration, M. Ackermann et al., Phys.Rev.Lett. 107 (2011) 241302, arXiv:1108.3546 [astro-ph.HE].

[36] L. Bergstrom, P. Ullio, and J. H. Buckley, Astropart.Phys. 9 (1998) 137-162, arXiv:astro-ph/9712318 [astro-ph]. 